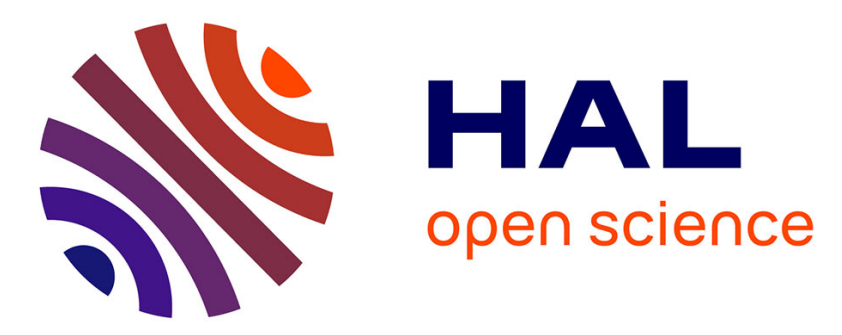

\title{
Finding approximate repetitions under Hamming distance
}

Roman Kolpakov, Gregory Kucherov

\section{To cite this version:}

Roman Kolpakov, Gregory Kucherov. Finding approximate repetitions under Hamming distance.

[Research Report] RR-4163, INRIA. 2001, pp.30. inria-00072459

\section{HAL Id: inria-00072459 \\ https://hal.inria.fr/inria-00072459}

Submitted on 24 May 2006

HAL is a multi-disciplinary open access archive for the deposit and dissemination of scientific research documents, whether they are published or not. The documents may come from teaching and research institutions in France or abroad, or from public or private research centers.
L'archive ouverte pluridisciplinaire HAL, est destinée au dépôt et à la diffusion de documents scientifiques de niveau recherche, publiés ou non, émanant des établissements d'enseignement et de recherche français ou étrangers, des laboratoires publics ou privés. 


\section{N R I A}

INSTITUT NATIONAL DE RECHERCHE EN INFORMATIQUE ET EN AUTOMATIQUE

\section{Finding approximate repetitions under Hamming distance}

Roman Kolpakov and Gregory Kucherov

$N^{\circ} 4163$

Avril 2001

THÈME 2 



\title{
RINRIA
}

\section{Finding approximate repetitions under Hamming distance}

\author{
Roman Kolpakov* and Gregory Kucherov \\ Thème 2 - Génie logiciel \\ et calcul symbolique \\ Projet Adage
}

Rapport de recherche $\mathrm{n}^{\circ} 4163$ - Avril 2001 - 30 pages

\begin{abstract}
The problem of computing tandem repetitions with $K$ possible mismatches is studied. Two main definitions are considered, and for both of them an $O(n K \log K+S)$ algorithm is proposed ( $S$ the size of the output). This improves, in particular, the bound obtained in [LS93]. Finally, other possible definions are briefly analyzed.
\end{abstract}

Key-words: Algorithm, pattern matching, tandem repeat, approximate repetition

* on leave from the French-Russian Institute for Informatics and Applied Mathematics at Moscow University 


\section{Recherche de répétitions approchées sous la distance de Hamming}

Résumé : Nous étudions le problème de recherche de répétitions en tandem avec $K$ inégalités. Nous considérons deux définitions principales et pour chacune d'elles nous proposons un algorithme de recherche s'exécutant en temps $O(n K \log K+S)$, où $n$ est la longueur du mot et $S$ est la taille de sortie. Cela améliore en particulier l'algorithme proposé dans [LS93]. Dans la dernière section nous analysons d'autres définitions possibles.

Mots-clés : Algorithme, recherche de motifs, répétition en tandem, répétition approchée 


\title{
Finding approximate repetitions under Hamming distance
}

\author{
Roman Kolpakov and Gregory Kucherov \\ LORIA/INRIA-Lorraine \\ 615, rue du Jardin Botanique \\ B.P. 101 \\ 54602 Villers-lès-Nancy France \\ e-mail: \{roman, kucherov\}@loria.fr
}

\section{Introduction}

Repetitions (periodicities) play a central role in word combinatorics [Lot83, CK97]. On the other hand, repetitions are important from the application perspective. As an example, their properties allow to speed up pattern matching algorithms [GS83, CR95, CH98].

The problem of efficiently identifying repetitions in a given word is one of the classical pattern matching problems [Cro81, Sli83]. A tandem repeat or a square is a pair of consecutive occurrences of a subword in a word. For example, $b a b a$ is a tandem repeat in word cbacbabacba. Since the beginning of 80s [Cro83] it is known that checking whether a word contains no tandem repeat (or is square-free) can be done in time $O(n)$ ( $n$ length of the word). If one wants to find all tandem repeats, their number comes into consideration. Word $a^{n}$ contains $O\left(n^{2}\right)$ tandem repeats. If we restrict ourselves to primitive

${ }^{*}$ on leave from the French-Russian Institute for Informatics and Applied Mathematics at Moscow University

RR $\mathrm{n}^{\circ} 4163$ 
squares (i.e. subwords $u u$ where $u$ is not itself a repetition $v^{k}$ for $k \geq 2$ ), then a word may contain $O(n \log n)$ of them and this bound is tight. All primitive squares can be found in time $O(n+S)$ where $S$ is their number [Kos94, SG98, KK99a], hence in the worst-case time $O(n \log n)$.

In [KK99b, KK99a], we studied maximal repetitions (see also [ML84, Mai89]). Those can be viewed as maximal runs of squares [IMS97, SG98], i.e. series of squares of equal length shifted by one letter one with respect to another. For example, bcbacacacaab contains a maximal repetition acacaca which is a succession of four squares : acac, caca, acac, caca. Thus, the set of maximal repetitions can be regarded as an encoding of all tandem repeats in the string. We showed [KK99b] that this encoding is more compact in the worst case, as there are only $O(n)$ maximal repetitions in words of length $n$. Moreover, all of them can be found in time $O(n)$ [KK99a].

More recently, searching for repetitions in a string received a new motivation, due to the biosequence analysis [Gus97]. Successive occurrences of a fragment often bear important information in DNA sequences and their presence is characteristic for many genomic structures (such as telomer regions for example). From practical viewpoint, satellites and alu-repeats are involved in chromosome analysis and genotyping, and thus are of great interest to genomic researchers. Tools for finding successive repeats are nowadays an obligatory part of integrated systems for analyzing and annotating whole genomes [Ben99]. We refer to [vBSvL ${ }^{+} 97$ ] as an example of biological study of contiguous repetitions in DNA sequences.

The major difficulty in finding biologically relevant repetitions in genomic sequences is a certain variation that must be admitted between the copies of the repeated subword. In other words, biologists are interested in approximate repetitions and not necessarily in exact repetitions only. The first natural definition of approximate repetition is an approximate tandem repeat which is a subword $u v$ where $u$ and $v$ are within a given distance $k$ and the notion of distance could be one of those usually used in biological applications, such as Hamming distance or edit distance. The problem of finding approximate tandem repeats for both these distances has been studied by G. Landau and J. Schmidt [LS93]. They showed that in case of the Hamming distance (respectively edit distance), all approximate tandem repeats can be found in time $O(n K \log (n / K)+S)$ (respectively $O(n K \log K \log n+S))$, where $S$ is the num- 
ber of repeats found. Several other approaches to finding approximate tandem repeats in DNA sequences have been proposed in bioinformatics community some of them use statistical framework [Ben98, Ben99], some require to specify the size of repeated motif [BW94, RDDD95], some use very general framework and have to make use of some heuristic filtering steps to avoid exponential blow-up [SM98].

This paper deals with finding approximate repetitions using exact combinatorial methods of string matching. We focus on the Hamming distance case when the variability between repeated copies can be only letter replacements. An important motivation is to define structures encoding families of approximate tandem repeats, analogous to maximal repetitions in the exact case. In Section 2, we define two fundamental structures : globally-defined approximate repetitions and runs of approximate tandem repeats. In Section 3, we show that all globally-defined approximate repetitions can be found in time $O(n K \log K+S)$, where $S$ is their number. In Section 4 we show that the same bound holds for runs of approximate tandem repeats: all of them can be found in time $O(n K \log K+R)$, where $R$ is their number. This result implies, in particular, that all approximate tandem repeats can be found in time $O(n K \log K+T)(T$ their number $)$, improving the $O(n K \log (n / K)+T)$ bound of G. Landau and J. Schmidt for the most interesting case of small $K$. Finally, in Section 5 we introduce two other possible notions of approximate repetitions and give a brief analysis of their properties.

\section{$2 \quad K$-mismatch globally-defined repetitions and runs of $K$-mismatch tandem repeats}

Quoting [Ben98], one difficulty in dealing with (approximate) tandem repeats is accurately defining them. Even if we concentrate only on mismatches, as it is the case in this paper, different definitions of approximate repetitions can be thought of. Here we introduce two basic notions of approximate repetitions. Other definitions will be discussed in Section 5 .

We start by recalling briefly some facts about exact repetitions. The period of a word $w[1: n]$ is the minimal natural number $p$ such that $w[i]=w[i+p]$ for all $1 \leq i, i+p \leq n$. The ratio $n / p$ is called the exponent of $w$. A repetition 
is any word with the exponent greater or equal to 2 [KK99b]. A tandem repeat, or a square, is a word which is a catenation of another word with itself. Equivalently, a tandem repeat is a repetition the exponent of which is an even natural number. In the case when the exponent is equal to 2 , the tandem repeat (square) is called primitive. The following proposition is well-known (see [Lot83]).

Proposition $2.1 A$ word $r[1: n]$ is a repetition of period $p \leq n / 2$ if and only if one of the following conditions holds:

(i) $r[1 . . n-p]=r[p+1 . . n]$, and $p$ is the minimal number with this property,

(ii) any subword of $r$ of length $2 p$ is a tandem repeat, and $p$ is the minimal number with this property.

When considering repetitions as subwords of a bigger word, the notion of maximality turns out to be very useful: a repetition is maximal iff it cannot be extended (by one letter) to the right or left while keeping the same period. Formally, given a word $w[1: n]$ and a subword $w[i . . j]$ which is a repetition of exponent $e \geq 2$, this repetition is called maximal if the period of both $w[i . . j+1]$ (provided that $j<n$ ) and $w[i-1 . . j]$ (provided that $i>1$ ) is strictly larger than $e$. For example, acaabaababc contains repetition (tandem repeat) $a a b a a b$ which is not maximal, as the $a$ which follows it respects the periodicity. On the other hand, aabaaba occurs as a maximal repetition. Maximal repetitions were studied in [Mai89, KK99b, KK99a, SG98].

We now turn to defining approximate repetitions. Similar to the exact case, the basic notion here is the approximate tandem repeat. Assume $h(\cdot, \cdot)$ is the Hamming distance between two words of equal length, that is $h\left(w_{1}, w_{2}\right)$ is the number of mismatches (letter differences at corresponding positions) between $w_{1}$ and $w_{2}$. For example, $h($ baaacb, $b c a b c b)=2$.

Definition 2.2 A word $\alpha=\alpha^{\prime} \alpha^{\prime \prime}$, such that $\left|\alpha^{\prime}\right|=\left|\alpha^{\prime \prime}\right|$, is called a $K$ mismatch tandem repeat iff $h\left(\alpha^{\prime}, \alpha^{\prime \prime}\right) \leq K$. Reusing the terminology of the exact case [KK99a], we call number $p=\left|\alpha^{\prime}\right|=\left|\alpha^{\prime \prime}\right|$ the period of $\alpha$, and words $\alpha^{\prime}, \alpha^{\prime \prime}$ left and right root of $\alpha$ respectively.

We now want to define a more global structure which would be able to capture "long approximate periodicities", generalizing repetitons of arbitrary 
exponent in the exact case. As opposed to the exact case, Conditions (i)-(ii) of Proposition 2.1 generalize to different notions of approximate repetition. Condition (i) gives rise to the strongest of them:

Definition 2.3 A word $r[1: n]$ is called a $K$-mismatch globally-defined repetition of period $p, p \leq n / 2$, iff $h(r[1 . . n-p], r[p+1 . . n]) \leq K$.

Equivalently, $r[1: n]$ is a $K$-mismatch globally-defined repetition of period $p$, if the number of $i$ such that $r[i] \neq r[i+p]$ is at most $K$. For example, abaa abba cbbacb is a 2-mismatch globally-defined repetition of period 4. $a b c a b c a b c a b b a b c a b c a b c a b c$ is a 1-mismatch globally-defined repetition of period 3 but $a b c a b c a b c a b b a b c a b c a b c a b b$ is not.

Another viewpoint, expressed by Condition (ii) of Proposition 2.1, considers a repetition as an encoding of squares it contains [IMS97, SG98]. Projecting this to the approximate case, we come up with the notion of run of approximate tandem repeats:

Definition 2.4 A word $r[1: n]$ is called a run of $K$-mismatch tandem repeats of period $p, p \leq n / 2$, iff for every $i \in[1 . . n-2 p+1]$, subword $\alpha=r[i . . i+2 p-1]=$ $r[i . i+p-1] r[i+l . . i+2 p-1]$ is a $K$-mismatch tandem repeat of period $p$.

Similarly to the exact case, when we are looking for approximate repetitions occurring in a word, it is natural to consider maximal approximate repetitions. These are repetitions extended to the right and left as far as possible provided that the corresponding definition is still verified. Note that the notion of maximality applies to both definitions of approximate repetition considered above : in both cases we can extend a repetition to the right/left as long as the obtained subword remains a repetition according to the considered definition. Throughout this paper we will be always interested in maximal repetitions, without mentioning it explicitely. Note that for both notions of approximate repetitions defined above, the maximality requirement implies that if $w[i: j]$ is a repetition of period $p$ in $w[1: n]$, then $w[j+1] \neq w[j+1-p]$ (provided $j<n)$ and $w[i-1] \neq w[i-1+p]$ (provided $i>1)^{1}$. Furthermore, if $w[i: j]$ is a maximal globally-defined repetition, it contains exactly $K$ mismatches

\footnotetext{
${ }^{1}$ For one type of repetitions defined in Section 5 this will not be the case, and we will add this condition explicitely.
} 


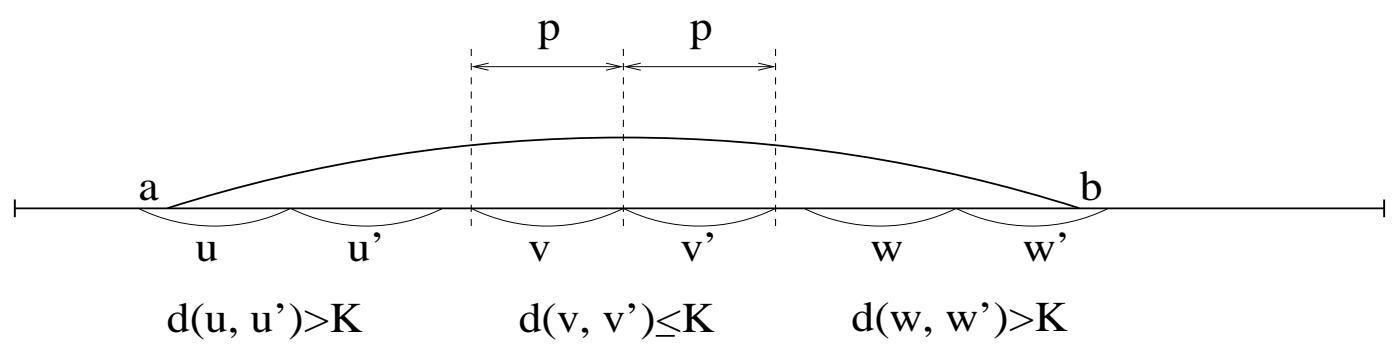

Figure 1: Maximal run of $K$-mismatch tandem repeats

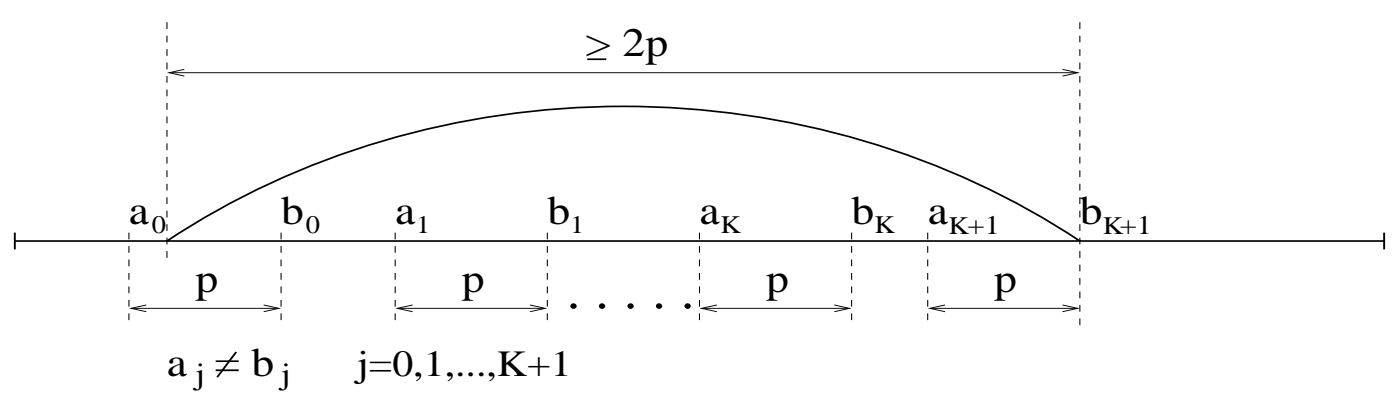

Figure 2: Maximal $K$-mismatch globally-defined repetition

$w[l] \neq w[l+p], i \leq l, l+p \leq j$, unless the whole word $w$ contains less than $K$ mismatches (to simplify the presentation, we always exclude this latter case from consideration).

Figure 1 illustrates the definition of (maximal) run of $K$-mismatch tandem repeats, and Figure 2 that of (maximal) $K$-mismatch globally-defined repetitions.

Example 2.5 The following Fibonacci word contains three runs of 3-mismatch tandem repeats of period 6 . They are shown in regular font, in positions aligned with their occurrences. Two of them are identical, and contain each four 3mismatch globally-defined repetitions, shown in italic for the first run only. The third run is a 3-mismatch globally-defined repetition in itself. 


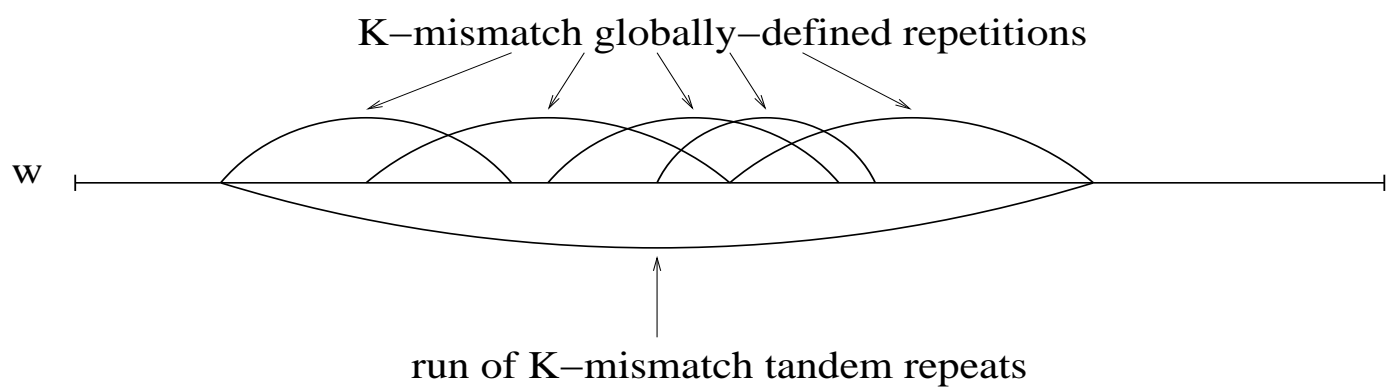

Figure 3: Extension relation

0100101001001010010100100101001001

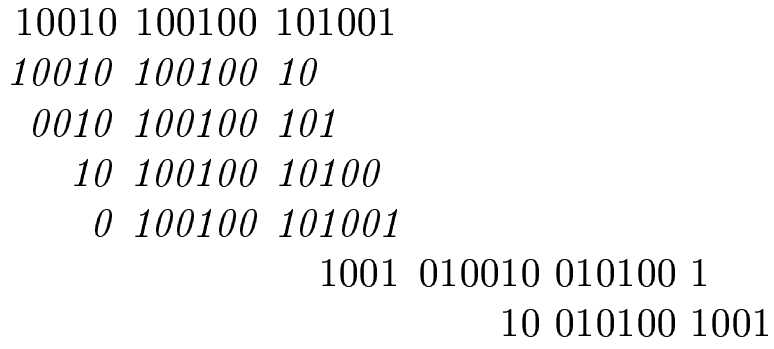

In general, each $K$-mismatch globally-defined repetition is a subword of a run of $K$-mismatch tandem repeats. On the other hand, a run of tandem repeats in a word is the union of all globally-defined repetitions it contains. We say then that the notion of run of $K$-mismatch tandem repeats extends that of $K$-mismatch globally-defined repetition (see Figure 3 ).

However, a run of tandem repeats may contain as many as a linear number of globally-defined repetitions. For example, the word $(000100)^{n}$ of length $6 n$ is a run of 1-mismatch tandem repeats of period 3 , which contains $(2 n-1)$ 1-mismatch globally-defined repetitions. Below is another example.

Example 2.6 The following run of 1-mismatch tandem repeats of period 4 contains 8 1-mismatch globally-defined repetitions, shown below in positions 
aligned with their occurrences.

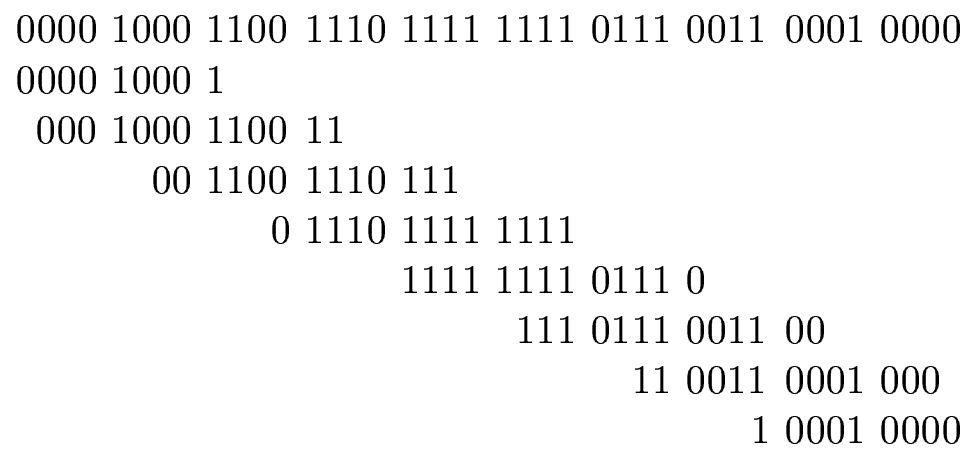

It is easily seen that the run can be iterated and therefore this gives another example of a family of runs containing a linear number of globally-defined repetitions.

In general, the following observation holds.

Lemma 2.7 Let $w[1: n]$ be a run of $K$-mismatch tendem repeats of period $p$ and let $s$ be the number of mismatches $w[i] \neq w[i+p], 1 \leq i, i+p \leq n$ (equivalently, $s=h(w[1 . . n-p], w[p+1 . . n]))$. Then $w$ contains $s-K+1$ globally-defined repetitions.

Note that both definitions can be criticized as for their relevance to practical situations. An obvious property of runs, as shown in Example 2.6, is that the repeated pattern can change completely along the run regardless the value of

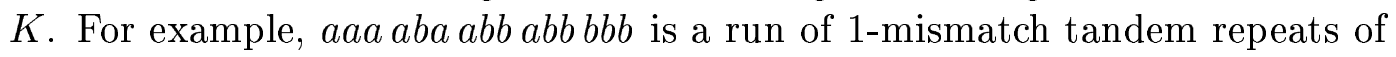
period 3, although 3-letter patterns $a a a$ and $b b b$ have nothing in common. On the other hand, globally-defined repetitions put a global limit on the number of mismatches and therefore may not capture some repetitions that one would possibly like to consider as such, in particular repetitions of big exponent where the total number of mismatches can exceed $K$ while the relative number of mismatches remains low. However, these two structures are of primary importance as they provide respectively the weekest and strongest notions of repetitions with $K$ mismatches, and therefore "embrace" all practically relevant repetitions. In what follows we propose efficient algorithms to find both those types of repetitions. 


\section{Finding $K$-mismatch globally-defined repeti- tions}

In this section we describe how to find, in a given word $w$, all maximal $K$ mismatch globally-defined repetitions occurring in $w$ ( $K$ is a given constant). Our algorithm extends, on the one hand, the one for exact maximal repetitions [Mai89, KK99a] and on the other hand, generalizes the one of [LS93] (see also [Gus97]) by using a special factorization of the word to speed-up the algorithm.

To proceed, we need more definitions. Consider a globally-defined repetition $r=w[i . . j]$ of period $p$ in a word $w[1: n] . w[i . . i+p-1]$ is called the left root of $r$ and $w[j-p+1 . . j]$ its right root. $r$ is said to contain the character $w[l]$ iff $i \leq l \leq j$, and is said to touch $w[l]$ iff $r$ contains $w[l]$, or contains one of characters $w[l-1], w[l+1]$.

We assume we fixed a minimal bound $p_{0}$ for the period of repetitions we are looking for. For example, $p_{0}$ can be taken to be $K+1$ having in mind that if a period $p \leq K$ is allowed, a tandem repeat of length $2 p$ with no common characters between the left and the right root would fall into the definition. This is purely pragmatic assumption which does not affect the method nor the complexity bounds.

Our first basic technique is described by the following auxiliary problem: Given a word $w[1: n]$ and a distinguished character $w[l], l \in[2 . . n-1]$, we wish to find all $K$-mismatch globally-defined repetitions in $w$ which touch $w[l]$. We distinguish two disjoint classes of repetitions according to whether their right root starts to the left or to the right to $w[l]$. We concentrate on repetitions of the first class, those of the second class are found similarly.

For each $p \in\left[p_{0} . . l-1\right]$, and for all $k \in[0 . . K]$, we compute the following functions :

$$
\begin{aligned}
& L P_{k}(p)=\max \{j \mid h(w[l-p . . l-p+j-1], w[l . . l+j-1]) \leq k\} \\
& L S_{k}(p)=\max \{j \mid h(w[l-p-j . . l-p-1], w[l-j . . l-1]) \leq k\}
\end{aligned}
$$

Informally, $L P_{k}(p)$ is the length of the longest subword in $w$ starting at position $l-p$ and equal, within $k$ mismatches, to a subword starting at $l$. Similarly, $L S_{k}(p)$ is the length of the longest subword ending at position $l-p-1$ equal, within $k$ mismatches, to a subword ending at position $l-1$. These functions 
are variants of longest common extension functions [LS93, Gus97] and can be computed in time $O(n K)$ using suffix trees combined with the lowest common ancestor computation in a tree. We refer to [Gus97] for a detailed description of the method.

Consider now a $K$-mismatch globally-defined repetition $r$ of period $p$ which has its right root starting to the left to $w[l]$. Note that character $w[l-p]$ is contained in $r$, and that $r$ is uniquely defined by the number of mismatches $w[i-p] \neq w[i], i \geq l$, contained in $r$. Let $k$ be the number of those mismatches. Then

$$
L P_{k}(p)+L S_{K-k}(p) \geq p .
$$

Conversly, (3) can be used to detect a repetition. The following theorem holds (see [LS93, Gus97]), which is a generalization of the corresponding result of [ML84, Mai89].

Theorem 3.1 Let $w[1: n]$ be a word and $w[l], 1<l<n$, a distinguished character. There exists a $K$-mismatch globally-defined repetition of period $p$ which contains $w[l]$, and has its right period starting to the left to $w[l]$, iff for some $k \in[0 . . K]$,

$$
L P_{k}(p) \leq p,
$$

and inequation (3) holds. In this case, this repetition starts at position $l-p-$ $L S_{K-k}(p)$ and ends at position $l+L P_{k}(p)-1$.

Inequation 4 ensures that the right root starts to the left of $w[l]$.

Theorem 3.1 provides an $O(n K)$ algorithm for finding all considered globallydefined repetitions: compute longest extension function (1) (2) (this takes time $O(n K))$ and then check inequations (3), (4) for all $p \in\left[p_{0} . . l-1\right]$ and all $k \in[0 . . K]$ (this takes time $O(n K)$ too). Each time the inequations are verified, a new repetition is identified. Finding repetitions with the right root starting to the right to $w[l]$ is a symmetric problem, which is solved within the same time bound.

The algorithm solving the auxiliary problem described above will be referred to as Algorithm 1. Its pseudo-code is shown below.

The second important tool is Lempel-Ziv factorization used in the wellknown compression method. Let $w$ be a word and assume that the last symbol of $w$ does not occur elsewhere. In this paper, we need two variants of 
$\overline{\text { Algorithm } 1 \text { Computing all } K \text {-mismatch globally-defined repetitions repeti- }}$ tions in $w$ touching a distinguished character

Input: word $w[1: n]$, position $l, 1<l<n$

Output: all $K$-mismatch globally-defined repetitions in $w$ which touch $w[l]$

\{Find those repetitions which have their right root starting to the left to $w[l]\}$

1: for all $p \in\left[p_{0} . . l-1\right], k \in[0 . . K]$, compute longest common extension functions $L P_{k}(p), L S_{k}(p)$ defined as in (1), (2)

2: for $p=p_{0}$ to $\min \{n-l+1, n / 2\}$ do

3: $\quad$ for $k=0$ to $K$ do

4: $\quad$ if $L P_{k}(p)+L S_{K-k}(p) \geq p$ and $L P_{k}(p) \leq p$ then

5: $\quad$ output a $K$-mismatch globally-defined repetition starting at position $l-p-L S_{K-k}(p)$ and ending at position $l+L P_{k}(p)-1$

\{Similarly, find those repetitions which have their right root starting to the right to $w[l]\}$

the Lempel-Ziv factorization, that we call with copy overlap and without copy overlap ${ }^{2}$.

Definition 3.2 The Lempel-Ziv factorization of $w$ with copy overlap (respectively without copy overlap) is the factorization $w=f_{1} f_{2} \ldots f_{m}$, where $f_{i}$ 's are defined inductively as follows:

- $f_{1}=w[1]$

- for $i \geq 2, f_{i}$ is the shortest word occurring in $w$ immediately after $f_{1} f_{2} \ldots f_{i-1}$ which does not occur in $f_{1} f_{2} \ldots f_{i}$ other than in prefix (respectively, does not occur in $\left.f_{1} f_{2} \ldots f_{i-1}\right)$.

As an example, the Lempel-Ziv factorization with copy overlap of the word $a a b b a b a b a b b b c$ is $a|a b| b a|b a b a b b| b c$; the factorization without copy overlap is

\footnotetext{
${ }^{2}$ The $s$-factorization used in [Mai89, KK99a] is a minor modification of the Lempel-Ziv factorization with copy overlap. The difference is that the $s$-factorization considers the longest factor occurring earlier, while the Lempel-Ziv factorization considers the shortest factor which does not occur earlier (see [Gus97] for a related discussion). In this paper, we use the Lempel-Ziv factorization which suits better to our purposes.
} 
$a|a b| b a|b a b| a b b b \mid c$. Both variants of Lempel-Ziv factorization can be computed in linear time [RPE81, Gus97]. If $w=f_{1} f_{2} \ldots f_{m}$ is the Lempel-Ziv factorization, we call $f_{i}$ 's Lempel-Ziv factors or simply factors of $w$. The last character of $f_{i}$ will be called the head of $f_{i}$.

We are now ready to describe the algorithm for finding all $K$-match globallydefined repetitions. Consider the Lempel-Ziv factorization of $w$ with copy overlap. The algorithm consists of three stages. The key to the first stage is the following lemmas.

Lemma 3.3 The right root of a $K$-mismatch globally-defined repetition cannot contain as subword $K+1$ consecutive Lempel-Ziv factors.

Proof: Each factor contained in the right root contains a character mismatching the one located one period to the left. Indeed, if it does not contain a mismatch, it has an exact copy occurring earlier, which contradicts to the definition of factorization. As the right root contains at most $K$ mismatches, it cannot contain $K+1$ or more factors.

We divide $w$ into consecutive blocks of $K+2$ Lempel-Ziv factors. Let $w=$ $B_{1} \ldots B_{m^{\prime}}$ be the partition of $w$ into such blocks. The last character of $B_{i}$ will be called the head character of this block. At the first stage, we find, for each block $B_{i}$, those repetitions which touch the head character of $B_{i}$ but does not touch that of $B_{i+1}$. First, concentrate on those of such repetitions with the right root starting before the head character of $B_{i}$.

Lemma 3.4 Assume a $K$-mismatch globally-defined repetition $r$ touches the head character of $B_{i}$ but not that of $B_{i+1}$. Then $|r|<2\left|B_{i} B_{i+1}\right|$.

Proof: Lemma 3.3 implies that the right root of $r$ cannot start before the first character of $B_{i}$. Therefore, the period of $r$ is bounded by $\left|B_{i} B_{i+1}\right|$. On the other hand, by the argument of the proof of Lemma 3.3, $r$ cannot extend by more than a period to the left of $B_{i}$. Therefore, the total length of $r$ is bounded by $2\left|B_{i} B_{i+1}\right|$.

Lemma 3.4 allows us to apply Algorithm 1 : Consider the word $w_{i}=v B_{i} B_{i+1}$, where $v$ is the suffix of $B_{1} \ldots B_{i-1}$ of length $\left|B_{i} B_{i+1}\right|$. Then find, using Algorithm 1 , all repetitions in $w_{i}$ touching the head character of $B_{i}$ and discard 
those which touch the head character of $B_{i+1}$. The resulting complexity is $O\left(K\left(\left|B_{i}\right|+\left|B_{i+1}\right|\right)\right)$.

After processing all blocks, we find all repetitions touching block head characters. Observe that repetitions resulting from processing different blocks are distinct. Summing up over all blocks, the resulting complexity of the first stage is $O(n K)$. The repetitions which remain to be found are those which lie entirely within a block - this is done at the next two stages.

At the second stage we find all repetitions inside each block $B_{i}$ which touch factor heads other than the block head (=last character of the block). For each $B_{i}$, we proceed by simple binary division approach:

(i) divide current block of factors $B=f_{i} f_{i+1} \ldots f_{i+m}$ into two sub-blocks $B^{\prime}=f_{i} \ldots f_{\lfloor m / 2\rfloor}$ and $B^{\prime \prime}=f_{\lfloor m / 2\rfloor+1} \ldots f_{i+m}$,

(ii) using Algorithm 1, find the repetitions in $B$ which touch the head character of $f_{\lfloor m / 2\rfloor}$, but discard those which touch the head character of $f_{i+m}$ or contain the first character of $f_{i}$,

(iii) process recursively $B^{\prime}$ and $B^{\prime \prime}$.

The above algorithm has $\lceil\log K\rceil$ levels of recursion, and since at each step the word is split into disjoint sub-blocks, the whole complexity of the second stage is $O(n K \log K)$.

Finally, at the third stage, it remains to find the repetitions which occur entirely inside each Lempel-Ziv factor, namely which don't contain its first character and don't touch its head character. By definition of factorization with copy overlap (Definition 3.2), each factor without its head character has another (possibly overlapping) occurrence to the left. Therefore, each of these repetitions has another occurrence to the left too. Using this observation, these repetitions can be found using the same technique as the one of [KK99a]: When constructing the Lempel-Ziv factorization we keep for each factor wa a pointer to a copy of $w$ to the left. Then processing factors from left to right, recover repetitions inside the factor from its pointed copy. We refer to [KK99a] for algorithmic details. The complexity of this stage is $O(n+S)$, where $S$ is the number of repetitions found.

The following theorem summarizes this section. 
Theorem 3.5 All K-mismatch globally-defined repetitions can be found in time $O(n K \log K+S)$ where $n$ is the word length and $S$ is the number of repetitions found.

The algorithm of finding all $K$-mismatch globally-defined repetitions, referred to as Algorithm 2, is given below.

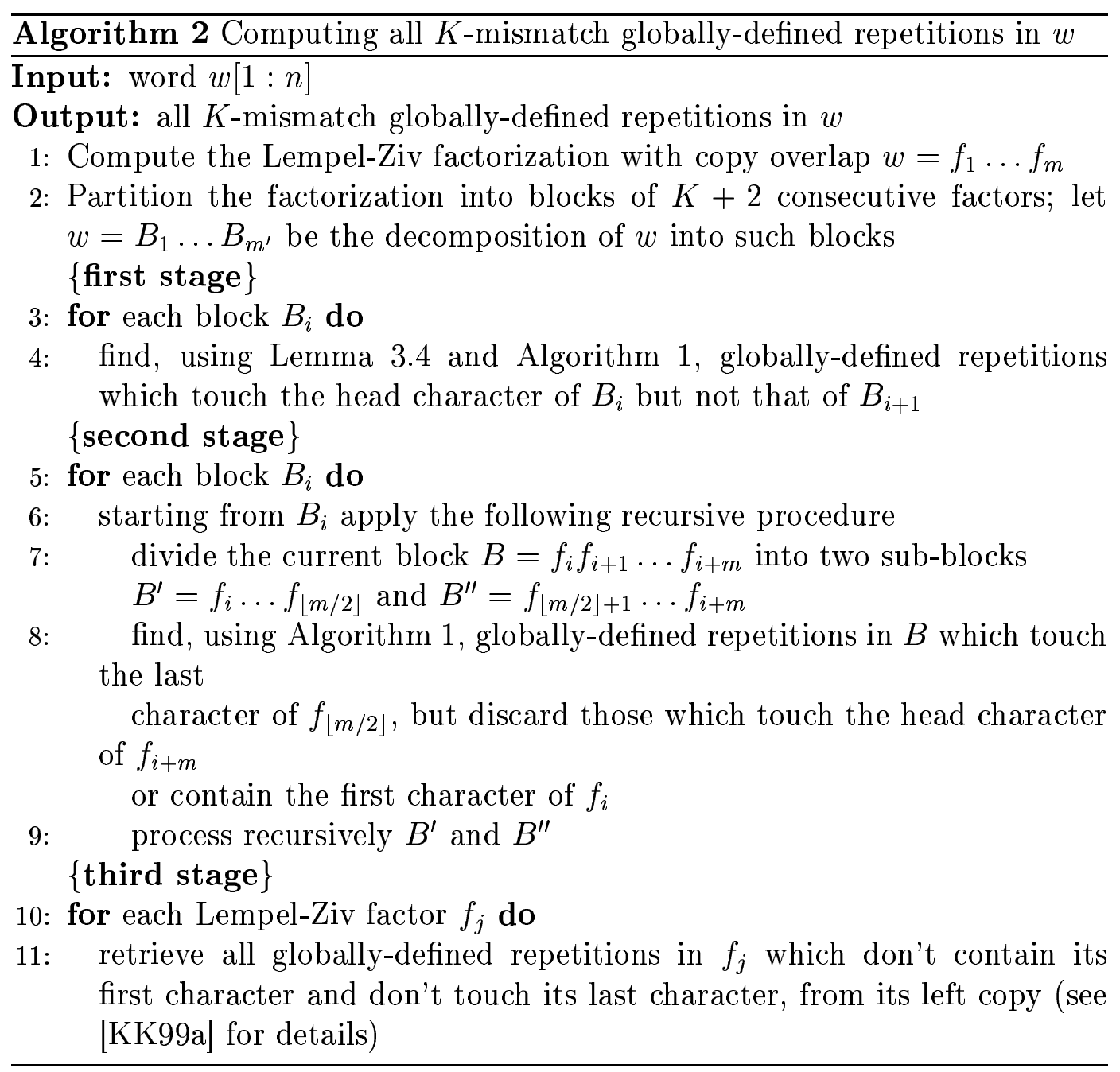




\section{Finding runs of $K$-mismatch tandem repeats}

In this section we discribe an algorithm for finding all runs of $K$-mismatch tandem repeats in a word.

The general structure of the algorithm is the same as for globally-defined repetitions (Algorithm 2) - it has the three stages playing similar roles. At the first and second stages, the key difference is the type of objects we are looking for : instead of computing globally-defined repetitions we now compute subruns of $K$-mismatch tandem repeats. Formally, a subrun is a run of $K$-mismatch tandem repeats, which is not necessarily maximal. At each point of the first and second stage when we search for repetitions touching some head character $w[l]$, we now compute subruns of those $K$-mismatch tandem repeats which touch $w[l]$. This can be seen as outputting by Algorithm 1 only the part of the globally-defined repetition falling to the interval $l-2 p . . l+2 p$. The modified Algorithm 1, referred to as Algorithm 3, is given below.

$\overline{\text { Algorithm } 3 \text { Computing subruns of } K \text {-mismatch tandem repeats in } w \text { touch- }}$ ing a distinguished character

Input: word $w[1: n]$, position $l, 1<l<n$

Output: all subruns of $K$-mismatch tandem repeats in $w$ which touch $w[l]$

\{Find those tandem repeats which have their right root starting to the left to $w[l]\}$

1: for all $p \in\left[p_{0} . . l-1\right], k \in[0 . . K]$, compute longest common extension functions $L P_{k}(p), L S_{k}(p)$ defined as in (1), (2)

2: for $p=p_{0}$ to $\min \{n-l+1, n / 2\}$ do

3: $\quad$ for $k=0$ to $K$ do

4: $\quad$ if $L P_{k}(p)+L S_{K-k}(p) \geq p$ and $L P_{k}(p) \leq p$ then

5: $\quad$ create a subrun of $K$-mismatch tandem repeats ending at positions $\operatorname{start}(p, k)=\max \left\{l+p-L S_{K-k}(p)-1, l-1\right\}$ through $\operatorname{end}(p, k)=$ $\min \left\{l+L P_{k}(p)-1, l+p-1\right\}$

6: $\quad$ if $k>0$ and $\operatorname{end}(p, k) \leq \operatorname{end}(p, k-1)+1$ then

7: $\quad$ merge this subrun with the subrun computed for the previous value of $k$

\{Similarly, find those tandem repeats which have their right root starting to the right to $w[l]$ and thus end in the interval $l+p . . l+2 p\}$ 
The major additional difficulty in computing runs is assembling subruns into runs. To perform the assembling, we need to store subruns in an additional data structure and to carefully manage merging of subruns into bigger runs. We have to ensure that the number of subruns we come up with and the work spent on processing them do not increase the resulting complexity bound.

The assembling occurs already at the level of Algorithm 3, as subruns found for different values of $k$ (for-loop at line 3) may overlap or immediately follow each other, in which case we join them into a bigger subrun (lines 6-7). Similarly, subruns of tandem repeats with the right root starting to the right to $w[l]$ (case non-shown in Algorithm 3) may have to be joined with subruns found by instructions 1-7 of Algorithm 3). We leave out the details of how this is done.

Below we describe the three stages of the algorithm in more details. We identify a subrun with the interval of end positions of the tandem repeats it contains.

For the input word $w$, we compute the Lempel-Ziv factorization without copy overlap and divide it into blocks $B_{1} \ldots B_{m^{\prime}}$, each containing $K+2$ consecutive Lempel-Ziv factors. At the first stage, we find subruns of all those tandem repeats which touch block head characters. For each block $B_{i}$, we find the tandem repeats which touch the head character of $B_{i}$ but not that of $B_{i+1}$. Let $l_{i}$ be the position of the head character of $B_{i}$. Then the subruns of period $p$, found at this step, belong to the interval $\left[l_{i}-1 . . \min \left\{l_{i}+2 p, l_{i+1}-2\right\}\right]$. We call this interval the explored interval for $w\left[l_{i}\right]$ and $p$. The subruns found at this step can be seen as subintervals of this explored interval. These subruns are stored into a double-linked list in increasing order of positions. (We leave it to the reader to check out that such a list can be easily computed by Algorithm 3 by making at each step a constant amount of extra work.) If the explored interval for $w\left[l_{i-1}\right]$ and $p$ ends at position $l_{i}-2$, it is merged with the explored interval for $w\left[l_{i}\right]$, thus forming a bigger explored interval. Accordingly, the lists of subruns associated with these intervals are merged into a single list. All additional operations take constant time, and the resulting complexity of the first stage is $O(n K)$.

The second stage is modified in a similar way. Recall that at each call of Algorithm 3 we are searching for repetitions occurring between some factor head, say $w\left[l^{\prime}\right]$, another factor head $w\left[l^{\prime \prime}\right]$, and touching some factor head $w[l]$ 
$\left(l^{\prime}<l<l^{\prime \prime}\right)$. Assuming that recursive calls are executed in preorder (see the description of the second stage in the previous section), no factor head between $w\left[l^{\prime}\right]$ and $w\left[l^{\prime \prime}\right]$ has been processed yet. In this case, the explored interval is $\left[\max \left\{l^{\prime}+2 p+1, l-1\right\} . . \min \left\{l+2 p, l^{\prime \prime}-2\right\}\right]$, and we may have to merge it either with the previous explored interval, or with the next one, or both. The complexity of the second stage stays $O(n K \log K)$.

After accomplishing the first and second stages, we have, for each period $p$, a set of non-intersecting explored intervals. Each interval is associated with a sequence of successive head characters $w\left[l_{i}\right], w\left[l_{i+1}\right], \ldots, w\left[l_{m}\right]$ such that $l_{j+1}-l_{j} \leq 2 p+2$ for $j \in[i . . m-1]$, and the interval itself is $\left[l_{i}-1 . . l_{m}+2 p\right]$. In particular, the interval is associated to $w\left[l_{i}\right]$ and $w\left[l_{m}\right]$ - the first and the last head characters of this sequence. Those subruns of tandem repeats which have been actually found within this interval, are stored in a double-linked list associated to the interval.

At the third stage, we have to find subruns of those tandem repeats which lie entirely inside Lempel-Ziv factors. For each period, potential occurrences of these subruns correspond precisely to the gaps between explored intervals. Thus, the third stage can be also seen as closing up the gaps between explored intervals for this period.

As in the previous section, the key observation here is the fact that LempelZiv factors without their head character have a copy to the left (here required to be non-overlapping), and the idea is again to process $w$ from left to right and to retrieve the subruns inside each factor from its copy. However, the situation here is different in comparison to globally-defined repetitions: we may have to "cut out" a chain of subruns belonging to the factor copy from a longer list and then to "fit" it into the gap between two explored intervals. The "cutting out" may entail splitting subruns which span over the borders of the factor copy, and "fitting into" may entail merging those subruns with subruns from the neighboring explored intervals. Below we sketch the algorithm for the third stage, which copes with these difficulties. Algorithm 4 given below provides a detailed description of the third stage.

During the computation of the Lempel-Ziv factorization, for each LempelZiv factor $f_{i}=v a$ we choose a copy of $v$ occurring earlier and point from the end position of this copy to the head character $a$ of $f_{i}$. It may happen that one position has to have several pointers, in which case we organize them in 


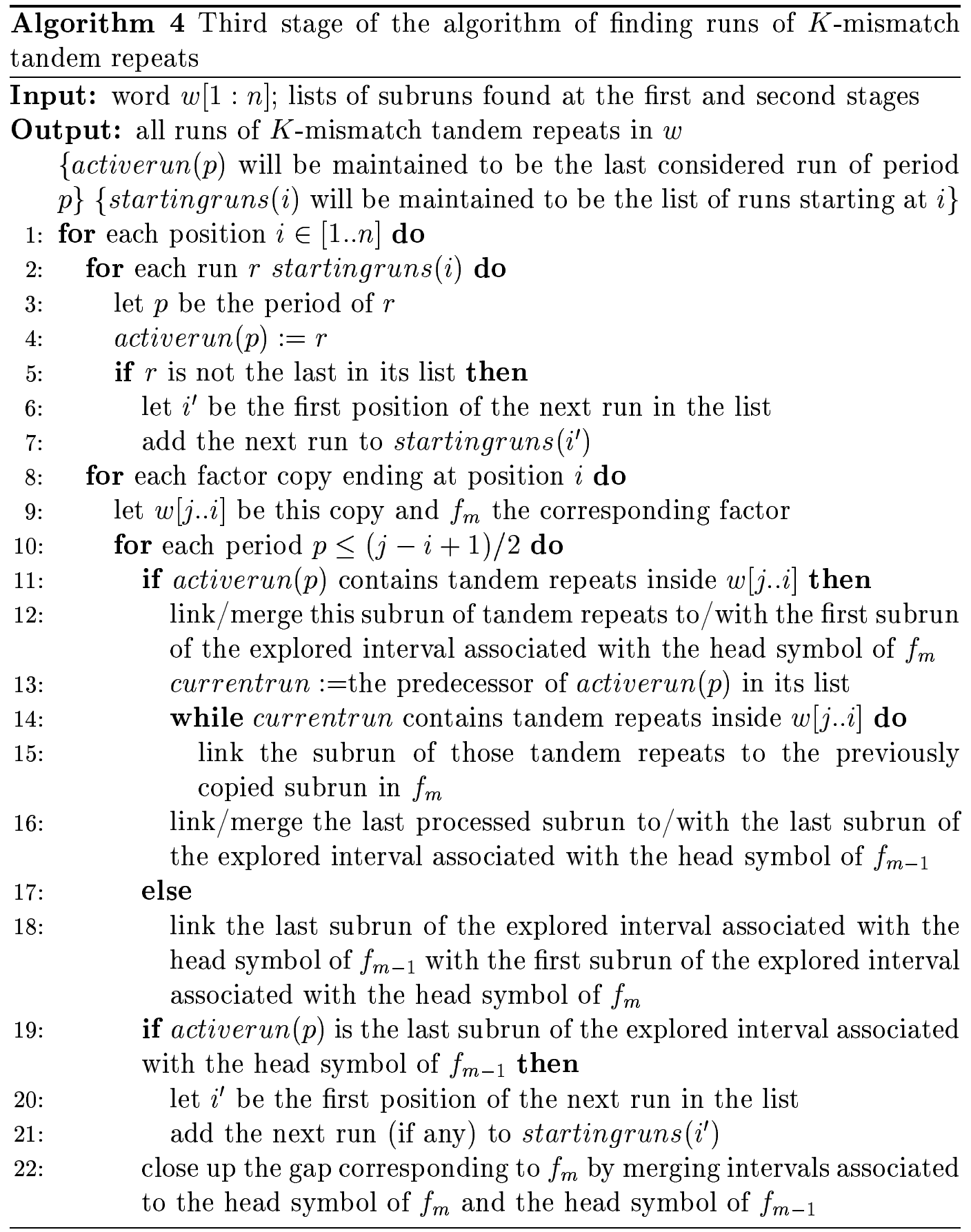


a list. We traverse $w$ from left to right and maintain for the current position the last runs (of all possible periods) which start before this character. To this purpose, we also maintain the following invariant: at the moment we arrive at a position, we know the list of all subruns which start at this position. This information is collected according to the following general rule: for each subrun starting at the current position, we assign the starting position of the next subrun in the list (instructions 2-7 in Algorithm 4). Of course, there may be no next subrun if the current subrun is the last one in the explored interval. In this case, the starting position of the subrun following the current subrun will be set at the moment we fill the gap after this explored interval (instructions 19-21).

When we arrive at the end position of a copy of a Lempel-Ziv factor, we need to copy into the factor all the subruns which this copy contains. Therefore, we scan backwards the subruns contained in the copy and copy them to the factor (instructions 11-18). Copying the subruns closes up two explored intervals into one interval, and links together two lists of subruns, possibly inserting a new list of runs in between. Copying subruns in the backward direction is important for the correction of the algorithm - this guarantees that no subruns are missed. It is also for these reason that we need the copy to be non-overlapping with the factor.

After the whole word has been traversed, no more gaps between explored intervals exist anymore. This means that for each period, we have a list of subruns with this period occurring in the word, which are actually the searched runs. The complexity of the third stage is $O(n+S)$, where $S$ is the number of resulting runs. Putting together the three stages, we obtain the main result of this section.

Theorem 4.1 All runs of $K$-mismatch tandem repeats can be found in time $O(n K \log K+S)$ where $n$ is the word length and $S$ is the number of runs found.

Once all runs have been found, we can easily output all tandem repeats. We then have the following result improving the result of [LS93].

Corollary 4.2 All K-mismatch tandem repeats can be found in time $O(n K \log K+S)$ where $n$ is the word length and $S$ is the number of tandem repeats found.

$\mathrm{RR} \mathrm{n}^{\circ} 4163$ 


\section{$5 \quad$ Other definitions of approximate repetitions}

$K$-mismatch globally-defined repetitions limit by $K$ the total number of mismatches, and therefore provide the strongest notion of approximate repetition. On the other hand, runs of $K$-mismatch tandem repeats provide the weekest notion of repetition with $K$ mismatches, as they impose only the minimal requirement that every tandem repeat in a such repetition contains no more than $K$ mismatches. For practical applications, such as genome analysis, it might be interesting to consider intermediate definitions with respect to the two "extreme" cases. In this section we introduce two such types of repetitions and point out very briefly some of their properties. A more detailed analysis, together with illustrating examples, is to appear in the extended version of this paper.

One natural way to loosen the definition of globally-defined repetitions is to limit by $K$ the Hamming distance between two subwords of length $p$ or less ( $p$ period), located within any distance which is a multiple of $p$.

Definition $5.1 A$ word $r[1: n]$ is called a $K$-mismatch uniform repetition of period $p, p \leq n / 2$ iff for every two subwords $r[i . i+j-1], r[i+k p . . i+k p+j-1]$ of $r$ such that $k$ is any integer and $1 \leq j \leq p$, we have $h(r[i . . i+j-1], r[i+$ $k p . . i+k p+j-1]) \leq K$.

For example, cbca bbca ab is a 1-mismatch uniform repetition of period 4, but $a b c a a b c a b c$ is not since $a b$ at position 1 is at Hamming distance 2 from $b c$ at position 9 . In general, $K$-mismatch uniform repetitions is a weaker notion than $K$-mismatch globally-defined repetitions: any word from $(a b c a d c)^{+}$is a 1-mismatch uniform repetition of period 3, whereas none of these words is a 1-mismatch globally-defined repetition.

The following technical remark concerning uniform repetitions is important. When we consider maximal uniform repetition in a word we have to add an additional condition: $w[i . . j]$ is a maximal $K$-mismatch uniform repetition of period $p$ in $w$ if neither $w[i-1 . . j]$ nor $w[i . . j+1]$ is a $K$-mismatch uniform repetition of period $p$ and the following inequalities hold:

$$
w[i-1] \neq w[i-1+p], w[j+1-p] \neq w[j+1]
$$




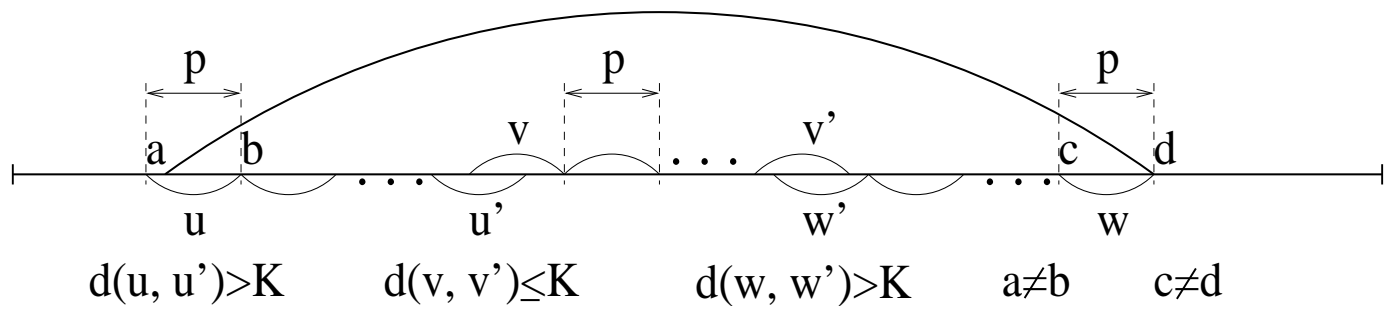

Figure 4: $K$-mismatch uniform repetition

In contrast to both notions of repetition considered before, inequalities (5) don't hold automatically for uniform repetitions and should be added explicitely in order to ensure, in particular, that every $K$-mismatch uniform repetition in a word is the union of $K$-mismatch globally-defined repetitions containing in it. The following example illustrates the situation.

Example 5.2 Consider the following word of length $4 p$. It contains two maximal $K$-mismatch globally-defined repetitions of period $p$ starting at position 1 and $p+1$ and shown below in regular font. Both of these repetitions are also maximal $K$-mismatch uniform repetitions. However, if we don't require inequalities (5) to hold, we obtain a series of $K-1$ "superfluous" $K$-mismatch uniform repetitions shown in italic.

$$
\begin{aligned}
& \overbrace{0 \ldots 00 \ldots 0}^{p} \overbrace{0 \ldots 0 \ldots 0}^{p-1} 1 \overbrace{0 \ldots 0}^{p-K} \overbrace{1 \ldots 1}^{K} \overbrace{0 \ldots 0}^{p-K-1} \overbrace{1 \ldots 111}^{K+1} \\
& 0 \ldots 00 \ldots 0 \quad 0 \ldots 0 \ldots 01 \quad 0 \ldots 01 \ldots 1 \quad 0 \ldots 0 \\
& \overbrace{0 \ldots 0}^{K-1} 0 \ldots 0 \ldots 010 \ldots 01 \ldots 10 \ldots 01 \\
& 0 \quad 0 \ldots 0 \ldots 010 \ldots 01 \ldots 10 \ldots 0 \overbrace{1 \ldots 1}^{K-1} \\
& 0 \ldots 0 \ldots 01 \quad 0 \ldots 01 \ldots 1 \quad 0 \ldots 01 \ldots 111
\end{aligned}
$$

Figure 4 gives an illustration to the definition of maximal $K$-mismatch uniform repetition. The relationship of uniform repetitions to globally-defined repetitions and runs are summarized in the following lemma. 
Lemma 5.3 (i) Any K-mismatch globally-defined repetition can be extended to a (possibly not unique) $K$-mismatch uniform repetition. Any $K$ mismatch uniform repetition is the union of $K$-mismatch globally-defined repetitions it contains.

(ii) Any K-mismatch uniform repetition can be extended to a unique run of $K$-mismatch tandem repeats. A run of $K$-mismatch tandem repeats is the union of $K$-mismatch uniform repetition it contains.

Another possible definition is obtained when one thinks about an approximate repetition as an "exact repetition with no more than $K$ replacement errors per period". This viewpoint is somewhat similar to the one of [SM98], where a repetition is defined through a consensus such that each repeated motif is within a specified distance from the consensus.

Definition 5.4 Word $r[1: n]$ is a $K$-mismatch consensus repetition of period $p, p \leq n / 2$, iff there exists an exact repetition $v[1: n]$ of period $p$ such that for any subword $r[i . . j]$ of $r$ such that $j-i \leq p$, we have $h(r[i . . j], v[i . . j]) \leq K$.

Example 5.5 Consider the word from Example 2.6. The 1-mismatch consensus repetitions it contains are shown together with a possible consensus for each of them shown below in italic.

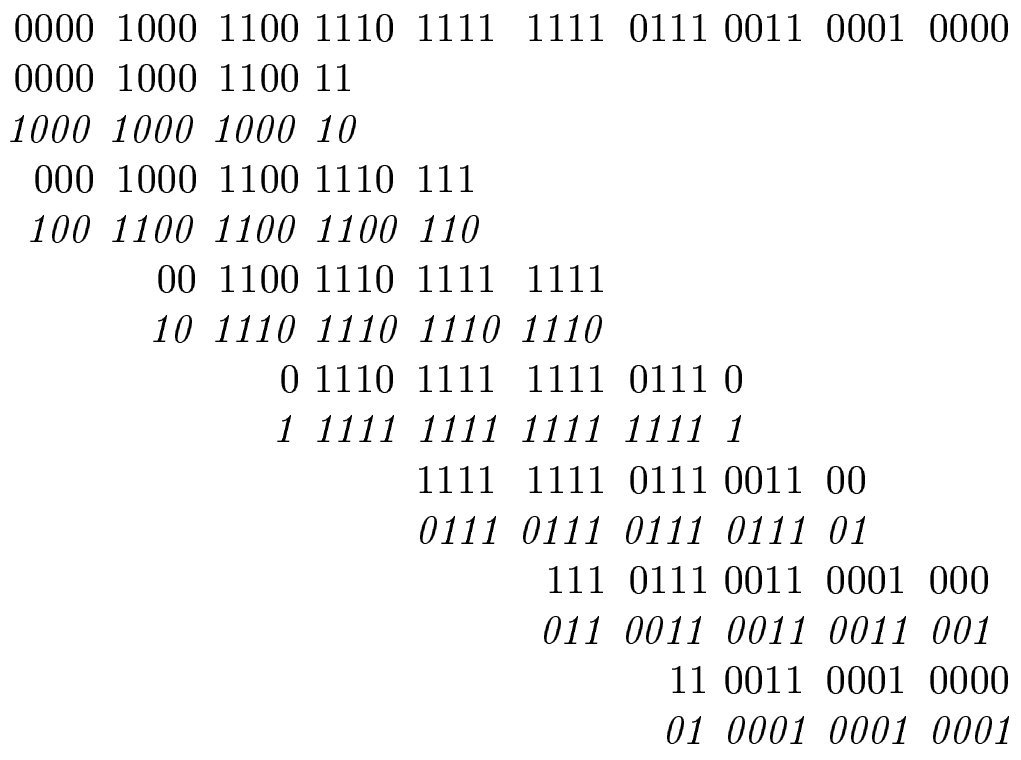




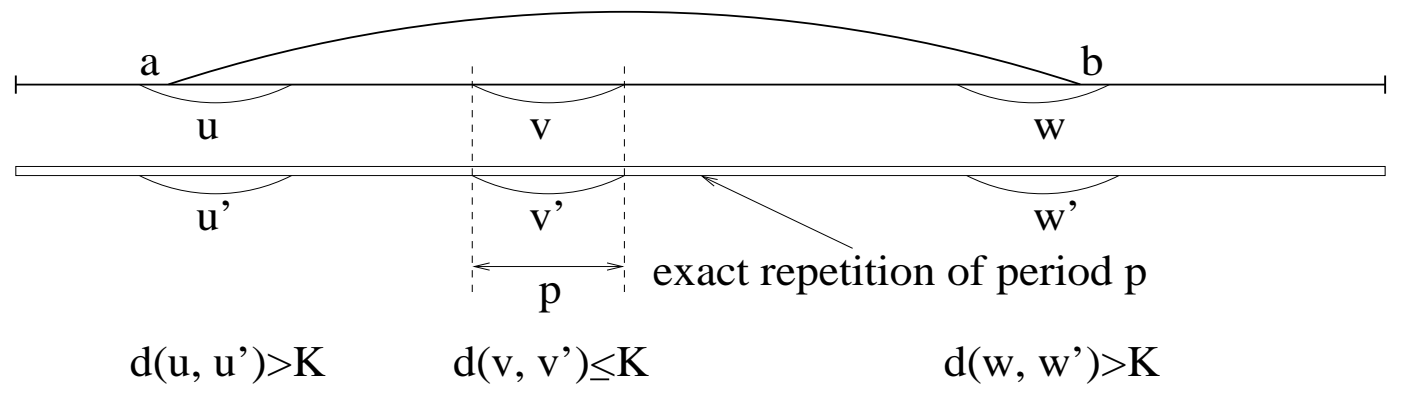

Figure 5: $K$-mismatch consensus repetition

The notion of $K$-mismatch consensus repetition is illustrated on Figure 5 . Similar to uniform repetitions, consensus repetitions provide an intermediate structure between globally-defined repetitions and runs:

Lemma 5.6 Assume $K$ is even.

(i) Any K-mismatch globally-defined repetition can be extended to a (possibly not unique) K/2-mismatch consensus repetition. Any K/2-mismatch consensus repetition is the union of $K$-mismatch globally-defined repetitions it contains.

(ii) Any K/2-mismatch consensus repetition can be extended to a unique run of $K$-mismatch tandem repeats. A run of $K$-mismatch tandem repeats is the union of $K / 2-$ mismatch consensus repetition it contains.

Concerning the relationship between uniform and consensus repetitions, it is easily seen that a $K / 2$-mismatch consensus repetition can be extended to a $K$-mismatch uniform repetition. Any $K$-mismatch uniform repetition can be, in turn, extended to a $K$-mismatch consensus repetition. More subtle relationship between these two notion require an additional analysis. Designing an efficient algorithm for finding these types of repetitions remains also an open question.

Relations between different notions of repetition, studied in this paper, is summarized on Figure 6. A solid arrow denotes the extension relation (see Figure 3 and remark after Example 2.5), and a flashed arrow means just that 


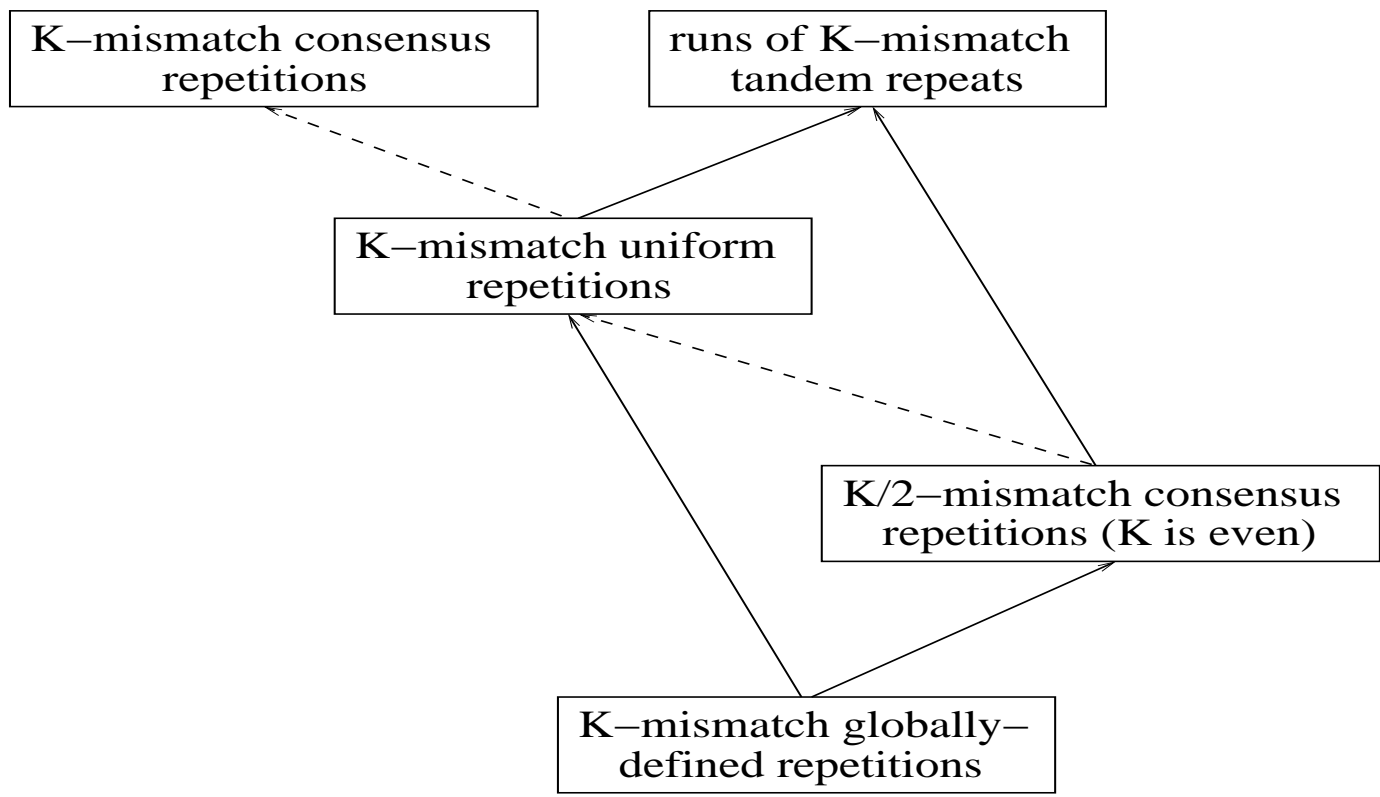

Figure 6: Relations between different notions of repetitions

the "source structure" can be extended to a "target structure", but not necessarily that a target structure is the union of the source structures contained in it.

\section{Concluding remarks}

We proposed $O(n K \log K+S)$ algorithms for finding $K$-mismatch globallydefined repetitions and runs of $K$-mismatch tandem repeats ( $S$ the output size). Note that if $K$ is considered constant, we have $O(n+S)$ algorithms for finding each of these structures. This is an interesting result, which has been long time unknown even for the exact case.

The algorithms presented in this paper are now being implemented within the mreps software ${ }^{3}$. Currently, mreps implements the algorithm of finding

\footnotetext{
${ }^{3}$ http://www. loria.fr/ ${ }^{\sim k u c h e r}$ ov/SOFTWARE/MREPS/index.html
} 
exact maximal repetitions [KK99a]. Some interesting experiments have been done by applying mreps to genomic sequences [GK00].

Acknowledgments We thank Mathieu Giraud, with whom we had first discussions on the subject. Early stages of this work have been supported by the REMAG project of INRIA.

\section{References}

[Ben98] G. Benson. An algorithm for finding tandem repeats of unspecified pattern size. In S. Istrail, P. Pevzner, and M. Waterman, editors, Proceedings of the 2nd Annual International Conference on Computational Molecular Biology (RECOMB 98), pages 2029. ACM Press, 1998.

[Ben99] G. Benson. Tandem repeats finder: a program to analyze DNA sequences. Nucleic Acids Research, 27(2):573-580, 1999.

[BW94] G. Benson and M. Waterman. A method for fast database search for all $k$-nucleotide repeats. Nucleic Acids Research, 22:48284836, 1994.

[CH98] R. Cole and R. Hariharan. Approximate string matching: A simpler faster algorithm. In Proceedings of the Ninth Annual ACMSIAM Symposium on Discrete Algorithms, pages 463-472, San Francisco, California, 25-27 January 1998.

[CK97] Ch. Choffrut and J. Karhumäki. Combinatorics of words. In G. Rozenberg and A. Salomaa, editors, Handbook on Formal Languages, volume I. Springer Verlag, Berlin-Heidelberg-New York, 1997.

[CR95] M. Crochemore and W. Rytter. Squares, cubes, and time-space efficient string searching. Algorithmica, 13:405-425, 1995. 
[Cro81] M. Crochemore. An optimal algorithm for computing the repetitions in a word. Information Processing Letters, 12:244-250, 1981.

[Cro83] M. Crochemore. Recherche linéaire d'un carré dans un mot. Comptes Rendus Acad. Sci. Paris Sér. I Math., 296:781-784, 1983.

[GK00] M. Giraud and G. Kucherov. Maximal repetitions and application to DNA sequences. In Proceedings of the Journées Ouvertes : Biologie, Informatique et Mathématiques, pages 165-172, Montpelier, 3-5 mai 2000 .

[GS83] Z. Galil and J. Seiferas. Time-space optimal string matching. Journal of Computer and System Sciences, 26(3):280-294, 1983.

[Gus97] D. Gusfield. Algorithms on Strings, Trees, and Sequences. Computer Science and Computational Biology. Cambridge University Press, 1997.

[IMS97] C.S. Iliopoulos, D. Moore, and W.F. Smyth. A characterization of the squares in a Fibonacci string. Theoretical Computer Science, 172:281-291, 1997.

[KK99a] R. Kolpakov and G. Kucherov. Finding maximal repetitions in a word in linear time. In Proceedings of the 1999 Symposium on Foundations of Computer Science, New York (USA). IEEE Computer Society, October 17-19 1999.

[KK99b] R. Kolpakov and G. Kucherov. On maximal repetitions in words. In Proceedings of the 12-th International Symposium on Fundamentals of Computation Theory, 1999, Iasi (Romania), Lecture Notes in Computer Science, August 30 - September 31999.

[Kos94] S. R. Kosaraju. Computation of squares in string. In M. Crochemore and D. Gusfield, editors, Proceedings of the 5th Annual Symposium on Combinatorial Pattern Matching, number 807 in Lecture Notes in Computer Science, pages 146-150. Springer Verlag, 1994. 
[Lot83] M. Lothaire. Combinatorics on Words, volume 17 of Encyclopedia of Mathematics and Its Applications. Addison Wesley, 1983.

[LS93] G. M. Landau and J. P. Schmidt. An algorithm for approximate tandem repeats. In A. Apostolico, M. Crochemore, Z. Galil, and U. Manber, editors, Proceedings of the 4th Annual Symposium on Combinatorial Pattern Matching, number 684 in Lecture Notes in Computer Science, pages 120-133, Padova, Italy, 1993. SpringerVerlag, Berlin.

[Mai89] M. G. Main. Detecting leftmost maximal periodicities. Discrete Applied Mathematics, 25:145-153, 1989.

[ML84] M.G. Main and R.J. Lorentz. An $O(n \log n)$ algorithm for finding all repetitions in a string. Journal of Algorithms, 5(3):422-432, 1984 .

[RDDD95] E. Rivals, O. Delgrange, J-P. Delahaye, and M. Dauchet. A first step towards chromosome analysis by compression algorithms. In N.G. Bourbakis, editor, Proceedings of the 1st IEEE Symposium on Intelligence in Neural and Biological Systems (INBS), Herndon, VA. IEEE Computer Society, May 29-31 1995.

[RPE81] M. Rodeh, V.R. Pratt, and S. Even. Linear algorithm for data compression via string matching. Journal of the ACM, 28(1):1624, Jan 1981.

[SG98] J. Stoye and D. Gusfield. Linear time algorithms for finding and representing all the tandem repeats in a string. Technical Report CSE-98-4, Computer Science Department, University of California, Davis, 1998.

[Sli83] A.O. Slisenko. Detection of periodicities and string matching in real time. Journal of Soviet Mathematics, 22:1316-1386, 1983.

[SM98] M.-F. Sagot and E.W. Myers. Identifying satellites in nucleic acid sequences. In S. Istrail, P. Pevzner, and M. Waterman, editors, Proceedings of the 2nd Annual International Conference on 
Computational Molecular Biology (RECOMB 98), pages 234-242. ACM Press, 1998.

[vBSvL ${ }^{+97}$ A. van Belkum, S. Scherer, W. van Leeuwen, D. Willemse, van Alphen L., and H. Verbrugh. Variable number of tandem repeats in clinical strains of haemophilus influenzae. Infect Immun, 65(12):5017-27, Dec 1997. 
Unité de recherche INRIA Lorraine

LORIA, Technopôle de Nancy-Brabois - Campus scientifique

615, rue du Jardin Botanique - BP 101 - 54602 Villers-lès-Nancy Cedex (France)

Unité de recherche INRIA Rennes : IRISA, Campus universitaire de Beaulieu - 35042 Rennes Cedex (France)

Unité de recherche INRIA Rhône-Alpes : 655, avenue de l'Europe - 38330 Montbonnot-St-Martin (France)

Unité de recherche INRIA Rocquencourt : Domaine de Voluceau - Rocquencourt - BP 105 - 78153 Le Chesnay Cedex (France)

Unité de recherche INRIA Sophia Antipolis : 2004, route des Lucioles - BP 93 - 06902 Sophia Antipolis Cedex (France) 